\title{
A THz Scanner Based on Planar Antenna-Supplied Silicon Field-Effect Transistors
}

\author{
D. Yavorskiy, ${ }^{1}$ J. Marczewski, ${ }^{2}$ K. Kucharski, ${ }^{2}$ P. Kopyt,${ }^{3}$ W. Gwarek, ${ }^{3}$ M. Ratajczyk, ${ }^{4}$ W. Knap,${ }^{5}$ B. Piętka, ${ }^{1}$ \\ and J. Łusakowski ${ }^{1}$ \\ ${ }^{I}$ Faculty of Physics, University of Warsaw, ul. Hoża 69, 00-681 Warsaw, Poland \\ ${ }^{2}$ Institute of Electron Technology, Al. Lotników 32/46, 02-668 Warsaw, Poland \\ ${ }^{3}$ Institute of Radioelectronics, ul. Nowowiejska 15/19, 00-665 Warsaw, Poland \\ ${ }^{4}$ VIGO System S.A., ul. Poznańska 129/133, 05-850 Ożarów Maz., Poland \\ ${ }^{5}$ TERALAB \& L2C CNRS, Universite Montpellier 2, 34-095 Montpellier, France
}

Received August 28, 2012; accepted September 28, 2012; published September 30, 2012

\begin{abstract}
A scanning device for imaging at THz frequencies was designed and constructed. The device comprised a $335 \sim \mathrm{GHz}$ source of radiation, an $\mathrm{x}-\mathrm{y}-\mathrm{z}$ translation stages, a number of optical elements and a detector. The detector was a silicon field-effect transistor supplied with a resonant antenna and a low-noise preamplifier. With a detector response of 10 V/W and a source power of $10 \sim \mathrm{mW}$ we could generate images in a DC mode with a speed limited only by the velocity of the stages movement.
\end{abstract}

In 2004, a new application was found for silicon metaloxide-semiconductor field-effect transistors (MOSFETs): it appeared that they can be used as room-temperature detectors of $\mathrm{THz}$ radiation [1]. Interesting in its own right, this fact would probably stay underestimated if it were not followed, two years later, by a much more sparkling result. The investigation of the responsivity of nanometer MOSFETs showed that their noise equivalent power places them among the best room temperature $\mathrm{THz}$ detectors [2]. This result could not have passed unnoticed because of expected profits resulting from the fabrication of new THz devices based on existing CMOS technology. In fact, a few academic, semi-industral and industral groups launched programs aiming at the construction of single detectors and matrices of detectors with $\mathrm{Si}$ MOSFETs as the basic units. It seemed that the fabrication of such a detector matrix was within easy reach. However, it was a much more complicated technology of microbolometer matrices that won the race to be the first commercially available $\mathrm{THz}$ camera [3].

On the MOSFET side, the leading group presented a focal-plane matrix composed of several pixels [4]. Although a single MOSFET pixel is typically less performant than a single microbolometer, there is a big advantage offered by a CMOS technology: a low price. The game is still on.

The physical mechanism lying behind the detection process is the rectification of high frequency plasma oscillations induced in the channel of a FET by incident radiation. Rectification occurs due to the asymmetry of a transistor channel and a nonlinear response of plasma to electromagnetic perturbation. The theory of the detection process was described in [5]. An electromagnetic wave is modelled as a high-frequency voltage source connected between the source and the gate of an FET and asymmetric boundary conditions at the source and drain side of the channel are assumed. A hydrodynamic approach is applied to describe the electron plasma motion with the Euler and continuity equations, and a linear dependence between the charge density and the electric potential is assumed - a relation valid in the case of FETs. The solution to this problem, to the second order of perturbation, shows that a constant photovoltage is created between the source and drain of an FET. This is a detection signal measured in experiments.

An important issue of any detector is the coupling with incident radiation. For detectors with a large aperture (e.g., Golay cells, pyroelectric detectors), the coupling is directly achieved by the absorption of photons in the detector. In the case of transistors, which dimension are much smaller than the wavelength of $\mathrm{THz}$ radiation, the efficiency coupling must be increased by an appropriate antenna. According to the theory, the antenna should be connected between the source and the gate of an FET. A properly designed antenna allows to maximize the potential difference between the gate and the source, which means that oscillations of the electric field on these electrodes are shifted in phase by $\pi$.

The efficiency of coupling is also strongly modified by propagation of substrate modes. One should bear in mind that at $\mathrm{THz}$ frequencies a semiconductor is practically a lossless material and a substrate plays the role of a resonator where the electromagnetic field is trapped [6]. 
This means that the semiconductor substrate constitutes a part of antenna which presence cannot be neglected in a detector's design. This is of particular importance in the case of multipixel structures in which the response of a pixel depends not only on its properties but also on the place where it is situated. A typical solution to the problem of substrate modes is to decrease the thickness of the substrate to a dimension which does not allow the propagation of $\mathrm{THz}$ modes, typically to a few tens of $\mu \mathrm{m}$.

The most important part of the project was to design and fabricate a detector which consisted of a Si-MOSFET and a low-noise preamplifier. The antenna design is shown in Fig. 1. A typical length of the channel was $5-10 \mu \mathrm{m}$, and the width $-10-20 \mu \mathrm{m}$. The transistors (n-type MOSFETs) were fabricated within a $3 \mu \mathrm{m}$ CMOS technology. The SOI (silicon-on-insulator) substrate was locally thinned down to $40 \mu \mathrm{m}$ by selective etching in $\mathrm{KOH}$. The transistors were subsequently processed over the thinned parts of the wafer. A polysilicon gate was used and the oxide thickness was about $60 \mathrm{~nm}$.

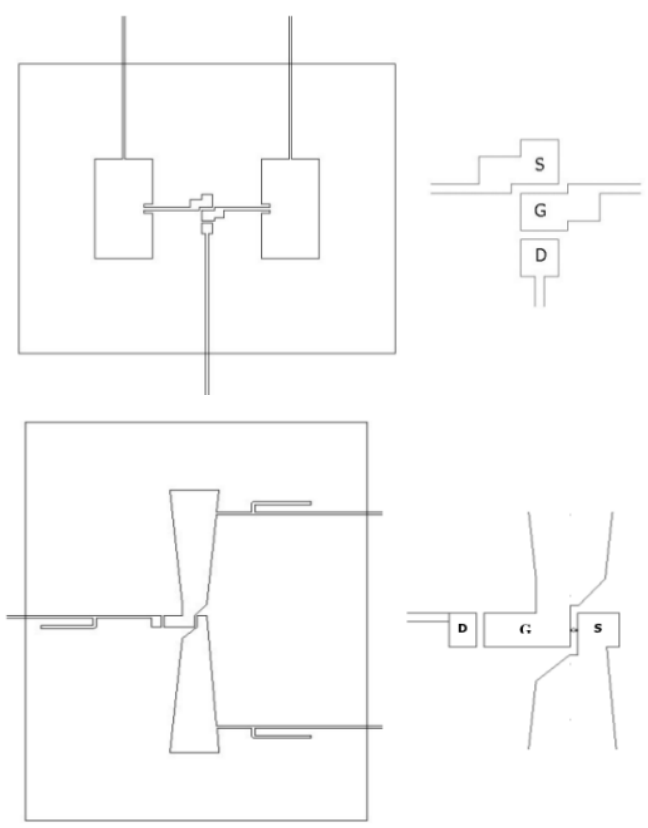

Fig. 1 Scheme of a patch antenna (upper graph) and a dipole antenna (lower graph) connected between source (S) and gate $(G)$. D is a drain contact.

The MOSFETs fabricated showed the threshold voltage $\mathrm{V}_{\mathrm{th}} \sim 1 \mathrm{~V}$ (Fig. 2, right scale). Transistors were exposed to $335 \mathrm{GHz}$ radiation from an electronic source based on a frequency multiplication chain, produced by Virginia Diodes Inc. The power of the source was equal to about $10 \mathrm{~mW}$ and the beam divergence was about $15^{\circ}$. The beam was reflected from an off-axis parabolic mirror and focused on a transistor with a teflon lens. The diameter of the focal point was equal to about $3 \mathrm{~mm}$. The electric circuit consisted of a voltage source for the gate-source polarization and a lock-in which measured the drainsource photovoltaic (PV) signal. The source of the FET was always grounded. The PV signal was measured as a function of gate polarization (see Fig. 2, left scale).

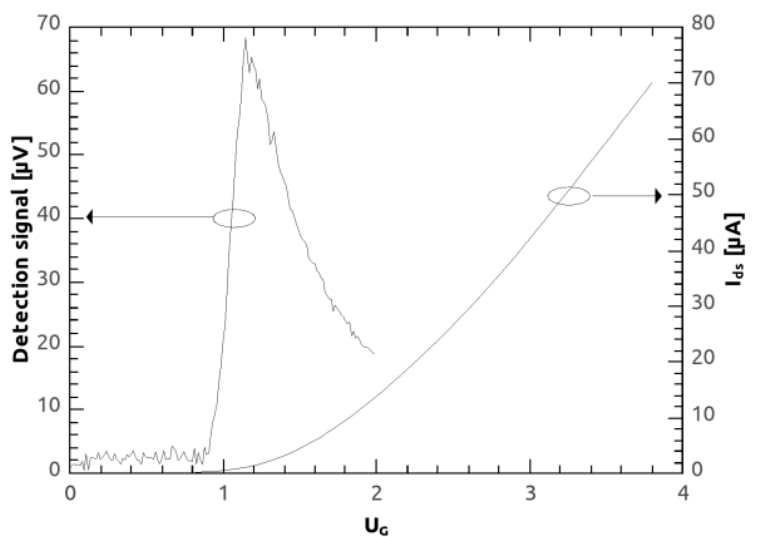

Fig. 2. Left scale: an example of the dependence of a photovoltaic signal on gate voltage. Right scale: a transfer characteristics.

A decrease and vanishing of the signal at voltages smaller than $\mathrm{V}_{\text {th }}$ is caused by shorting the photovoltaic signal with the input impedance of the lock-in [6]. The frequency of chopping the laser beam had to be adjusted carefully. On the one hand, it could not be too low because of the lockin network filters set for $50 \mathrm{~Hz}$ and $100 \mathrm{~Hz}$. On the other, it could not be too high because of an RC constant of the circuit. We have found that the maximum signal was obtained with a chopping frequency of about $200 \mathrm{~Hz}$. A maximum signal obtained in the described system was on a level of $0.5 \mathrm{mV}$. We could also use a low-noise preamplfier designed to match the parameters of transistors investigated. The amplification could be set between $10^{3}$ and $10^{4}$, which led to acquisition with a signal-to-noise ratio of about 5000.

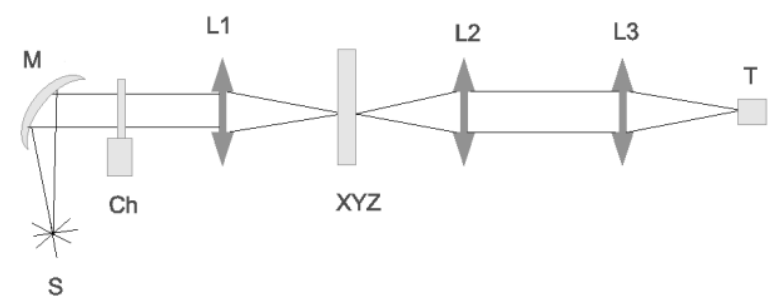

Fig. 3. Scheme of the scanner. L1, L2 and L3 are teflon lenses, M - offaxis parabolic mirror, $\mathrm{Ch}$ - chopper, $\mathrm{T}$ - transistor, $\mathrm{XYZ}$ - translation stages.

The scheme of the scanner is shown in Fig. 3. The object to be investigated is attached to an $x-y-z$ translation stage and placed in the focal plane of lens L1. The beam passing the object is directed through two subsequent 
lenses to be focalized on a detector. The scanner is controlled by software which enables to move the object with a defined accuracy (not less than $0.25 \mathrm{~mm}$ ). Two scanning modes were tested. In the AC mode, the beam of radiation was chopped, the scanner stopped after every step to allow the acquisition system to read the signal a prescribed number of times and to calculate an average. This mode is useful when working with low-power sources or less transparent materials. In the DC mode, the scanner moved without stopping with maximum speed (of about $5 \mathrm{~cm} / \mathrm{s}$ ) and the signal was acquired every $0.25 \mathrm{~mm}$ of the travel. The DC mode could be applied in our system due to a high dynamic range of the acquisition system when a preamplifier was used. This high dynamic range is also profitable when the sources of radiation are much weaker than the one used in the present experiment. An example of scan is shown in Fig. 4.

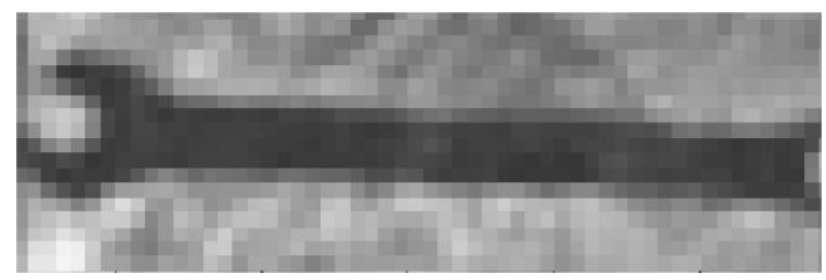

Fig. 4. Scan of a metallic tool hidden in a paper envelope.

In conclusion, planar antennas for Si-MOSFETs were designed and Si-MOSFETs were fabricated to be detectors of $335 \mathrm{GHz}$ radiation. A low-noise preamplifier allowed to construct an acquisition system with a signalto-noise ratio of about 5000. An x-y-z scanner was built, which enables to image objects in $\mathrm{AC}$ and $\mathrm{DC}$ acquisition modes with a spatial resolution of $0.25 \mathrm{~mm}$.

This work was supported by the National Center for Research and Development grant OR00015509 and by the Foundation for Polish Science POMOST/2010-1/8 program.

\section{References}

[1] W. Knap et al., Appl. Phys. Lett. 85, 675 (2004).

[2] R. Tauk et al., Appl. Phys. Lett. 89, 253511 (2006).

[3] N. Oda et al., Proc. SPIE 8012: doi:10.1117/12.888992.

[4] E. Ojefors, U. Pfeiffer, A. Lisauskas, H. Roskos, IEEE J. Solid-state Circuits 44, 1968 (2009).

[5] M. Dyakonov, M. Shur, IEEE Trans. on Electron Devices 43, 380 (1996).

[6] P. Kopyt, J. Marczewski, K. Kucharski, J. Łusakowski, W. Gwarek, Proc. 36th Conference on Infrared, Millimeter and Terahertz Waves, Huston, DOI: 10.1109/irmmw-THz.2011.6105129, ISBN: 978-1-45770510-6.

[7] M. Sakowicz et al., J. Appl. Phys. 110, 054512 (2011). 